\title{
Grupos Educativos e a Saúde de Pessoas que Vivem com Doenças Crônicas Não Transmissíveis
}

\section{Educational Groups and the Health of Patients Living with Chronic Noncomminucable Diseases}

\author{
Érika Monteiro da Silva' \\ Vera Maria Sabóia² \\ Lina Márcia Miguéis Berardinelli ${ }^{3}$ \\ Irma da Silva Brito ${ }^{4}$ \\ Joziane Santos da Silva ${ }^{5}$
}

${ }^{1}$ Enfermeira, Mestranda do Programa de Pós-Graduação em Enfermagem, Universidade Federal Fluminense, Especialista em Saúde Coletiva. E-mail: erika.rph@gmail.com

${ }^{2}$ Enfermeira, Pós-Doutora em Enfermagem, professora Titular da Disciplina de Fundamentos de Enfermagem da Escola de Enfermagem da Universidade Federal Fluminense. E-mail: verasaboia@uol.com.br

${ }^{3}$ Enfermeira, Pós-Doutora em Enfermagem, professora Associada do Departamento Enfermagem Médico Cirúrgico da Universidade do Estado do Rio de Janeiro. E-mail:I.m.b@uol.com.br

${ }^{4}$ Enfermeira, Pós-Doutora em Enfermagem, professora Adjunta do Departamento de Enfermagem Saúde Pública, Familiar e Comunitária da Escola Superior de enfermagem de Coimbra. E-mail: irmabrito@esenfc.pt

${ }^{5}$ Enfermeira, Especialista em Saúde do Idoso, Residente em Saúde Coletiva na Universidade Federal Fluminense. E-mail: jozysilva78@gmail.com

*Manuscrito baseado na Dissertação, intitulada: "Interdisciplinaridade na produção do cuidado no contexto da promoção da saúde", da Universidade Federal Fluminense, no ano de 2018. Mestranda: Érika Monteiro da Silva. Orientação: Profa Dra Vera Maria Sabóia 


\title{
Resumo
}

Objetiva-se apresentar as atividades educativas dos grupos de pessoas que vivem com DCNT e como os mesmos enfrentam a patologia. Trata-se de uma revisão integrativa, com busca nas bases de dados da BVS, PUBMED E MEDLINE, com a questão de pesquisa: quais estudos publicados em periódicos científicos da área de saúde no período de 2012 a 2016 sobre a repercussão de grupos educativos em saúde com pessoas que vivem com DCNT, visando a aplicabilidade dos achados na prática? Foram incluídas publicações disponíveis na íntegra, em português, inglês e espanhol, indexadas nas referidas bases de dados. Foram selecionados 5 artigos e analisados em duas categorias: "A educação em saúde como forma de reorientação de hábitos de vida" e "Desafios na busca da superação do modelo biomédico". A realização de grupos educativos como forma de promoção da saúde ainda necessita ser aprimorada. Compreende-se novas intervenções adequadas à realidade das pessoas com DCNT, considerando aspectos de vida e sociedade, visando sua autonomia.

Palavras-chave: Doença Crônica; Educação em Saúde; Promoção da Saúde; Estrutura de Grupo.

\begin{abstract}
This study aims to explore publications on the educational activities of the groups of people living with Chronic NonCommunicable Diseases (NCDs) and how they face the pathology. It is an integrative review based on data taken from BVS, PUBMED and MEDLINE. Research question: Which studies regarding the repercussion of educational groups focused on the healthcare of people who live with NCDs were published in scientific health journals between 2012 and 2016, aiming their practical applicability? Publications that can be found online were attached to this work's database. The five selected articles were analyzed and divided in two categories: The promotion of healthcare education as a way of reorienting individuals' lifestyles and the challenges in the pursuit of overcoming the biomedical model that is popular nowadays. The organization of educational groups for the purpose of promoting healthcare still needs to be improved. It is acknowledged that new interventions, concerning the autonomy of individuals with NCDs are more appropriate to their reality.
\end{abstract}

Keywords: Chronic diseases, healthcare education, healthcare promotion, group structure.

\section{Introdução}

O Instituto Brasileiro de Geografia e Estatística (IBGE) $^{(1)}$ estima que o Brasil terá mais de 32 milhões de pessoas com 60 anos ou mais em 2025, o que representará $10,7 \%$ da população. Assim, a população idosa brasileira será a sexta maior do mundo.

Com a transição demográfica, ocorre também uma mudança na área epidemiológica, ou seja, há uma ascensão das doenças crônicas não transmissíveis (DCNT), já que a população idosa é mais propensa ao desenvolvimento dessas doenças. Além disso, fatores como baixa escolaridade, tabagismo, maus hábitos alimentares, falta de atividade física e consumo de álcool estão associados ao aumento do número de pessoas com DCNT. Estes últimos estão ligados à epidemia de sobrepeso e obesidade e à elevada prevalência de hipertensão arterial e hipercolesterolemia(2).

Há registro de que, nos últimos anos, $63 \%$ das mortes no mundo foram em consequência de DCNT. No Brasil, essas doenças estão entre as principais causas de internações hospitalares e geram perda da qualidade de vida, alto grau de limitação nas atividades de trabalho e lazer, aumento no número de mortes prematuras, além de impactos econômicos para as famílias, comunidade e a sociedade em geral $^{(2)}$.

Entre as doenças que contribuem para a atual mudança no perfil de morbimortalidade da população brasileira destacam-se as a Hipertensão Arterial Sistêmica (HAS), o Diabetes Mellitus (DM), o câncer e as doenças respiratórias crônicas. No Brasil, as DCNT compõem um problema de saúde pública, sendo responsáveis por $72 \%$ das causas de morte. Destas, 31,3\% são doenças do aparelho circulatório $^{(3)}$.

As doenças cardiovasculares representavam somente $12 \%$ das mortes na década de 30 . Atualmente, são as principais causas de morte em todas as regiões brasileiras. Em segundo lugar, estão os cânceres, e em terceiro, as mortes ocasionadas por acidentes e violências ${ }^{(4)}$

A Hipertensão Arterial e o Diabetes Mellitus estão intimamente ligados às estatísticas, uma vez que pertencem ao grupo de doenças que causam lesões irreversíveis, incapacitantes, e a morte ${ }^{(5)}$.

Durante muitos anos, ter saúde significava apenas a ausência de doença, vivia-se num campo de 


\section{ARTIGO ORIGINAL}

prática voltada às ações curativas. Culturalmente isso ainda está embutido no cotidiano da população e dos profissionais de saúde, onde a procura pelos serviços de saúde é para aliviar uma necessidade de cuidados momentânea, numa concepção biomédica e formatada no processo saúde-doença ${ }^{(6)}$.

Há a necessidade de mudanças nas estratégias e ações de promoção da saúde de forma a levar a população a ser mais atuante no processo, estimulando adequações e reorientação de comportamentos e hábitos de vida. É indispensável realizar ações de educação em saúde pautadas na dialogicidade e nas necessidades do grupo populacional visando sua autonomia ${ }^{(6)}$.

Desta forma, é fundamental a perspectiva da construção do cuidado centrada nos usuários e suas necessidades, e não de um modelo que atenda aos interesses do mercado. Diante do atual cenário de transição epidemiológica e demográfica com a predominância das doenças crônicas não transmissiveis (DCNT) e a ampliação da população de idosos, há um aumento da demanda por agregação de tecnologias, o que torna indispensável repensar o modelo de assistência praticado, priorizando os atos cuidadores e a autonomia dos sujeitos ${ }^{(7)}$.

As Tecnologias Educativas em Saúde (TESs) são ferramentas importantes para o trabalho educativo no processo de cuidado. Através de grupos educação em saúde é possível favorecer a participação como forma de garantir ao indivíduo e à comunidade, a possibilidade de decidir sobre seus próprios destinos, e capacitar esses sujeitos para atuarem para melhorar sua saúde ${ }^{(8) .}$

Assim, a pergunta que norteou esta pesquisa, foi: Quais são as atividades educativas realizadas com grupos de pessoas que vivem com Hipertensão e Diabetes Mellitus para melhorar as condições de saúde dos participantes, assim como sua postura frente à doença?

Com o intuito de despertar outros estudos e motivar ações educativas com grupos de forma participativa, objetivou-se apresentar as atividades educativas dos grupos de pessoas que vivem com DCNT e como os mesmos enfrentam a patologia.

\section{Método}

Este estudo faz parte de um projeto internacional, multicêntrico entre Brasil e Portugal, proposto pela Faculdade de Enfermagem da Universidade do Estado do Rio de Janeiro (ENF/UERJ), entre a Escola de Enfermagem Aurora de Afonso Costa, da Universidade Federal Fluminense (EEAAC/UFF) e a Escola Superior de Enfermagem de Coimbra em Portugal (ESEC), com o tema: "O cuidado em saúde e enfermagem: práticas de saúde e prevenção de agravos no Município do Rio de Janeiro". O propósito desta parceria foi avaliar a utilidade do grupo de promoção da saúde na doença crônica na melhoria do autocuidado e potencialização da interdisciplinaridade em diferentes cenários. As ações desenvolvidas em grupo e as práticas de cuidado foram compartilhadas em conjunto com os enfermeiros do serviço, docentes, e estudantes do ensino de Graduação das três Universidades envolvidas no processo de construção dos estudos.

Trata-se de um estudo de Revisão Integrativa (RI), caracterizado pela busca de pesquisas disponíveis nas bases de dados LILACS, SCIELO, MEDLINE e PUBMED em base de dados científicos, com análises e discussões compatíveis. Faz parte de um recorte da dissertação de mestrado em ciências do cuidado em saúde da EEAAC/UFF com o título: "Interdisciplinaridade na produção do cuidado no contexto da promoção da saúde", do ano de 2018. A Rl permite reunir e sintetizar estudos referentes a um determinado tema, a fim de fornecer subsídios para reflexão e compreensão do que está sendo investigado, assim como sua aplicabilidade na prática ${ }^{(9-10)}$.

Seis etapas distintas são utilizadas para realização da revisão integrativa: elaboração da pergunta norteadora; busca ou amostragem na literatura; coleta de dados; análisecrítica dos estudos incluídos; discussão dos resultados; e apresentação da revisão integrativa ${ }^{(10)}$.

Os critérios de inclusão utilizados foram artigos disponíveis, publicados na íntegra em português, inglês e espanhol, indexados entre os anos de 2012 a 2016 e que abordassem o tema sobre atividades educativas realizadas com grupos de pessoas que 
vivem com DCNT, como Hipertensão e Diabetes Mellitus nas unidades de saúde. Estabeleceram-se como critério de exclusão: artigos repetidos nas bases virtuais; artigos em outras línguas; os que não abordavam a temática em questão; artigos que não estavam disponíveis na íntegra; e que sua abordagem não contribuía para o conhecimento na área pretendida.

Foi realizada busca nas bases virtuais utilizando os seguintes descritores: estrutura de grupo; doença crônica; educação em saúde e promoção da saúde. A fim de obter o maior número de artigos sobre a temática escolhida, foram elaboradas estratégias de busca por meio de combinações e utilização do operador booleano AND.

Primeiramente utilizou-se o cruzamento dos descritores doença crônica AND educação em saúde AND promoção da saúde. Assim, encontrouse 434 produções, sendo 94 artigos dentro dos critérios de inclusão pré-estabelecidos. Após leitura dos títulos foram selecionados 20 artigos, sendo que 2 foram excluídos por repetição, e após a leitura dos resumos, 4 artigos foram selecionados para leitura na íntegra.

Posteriormente, foram cruzados os descritores estrutura de grupo AND educação em saúde, sendo encontrados 4 artigos de acordo com os critérios, que após leitura dos títulos e resumos, 1 artigo foi selecionado para leitura integral.

\section{Resultados}

Após a busca, a amostra final de 5 artigos encontrados foram lidos na íntegra de maneira crítica e reflexiva, e separados de acordo com as seguintes informações: Título; Base de dados; Periódico (ano e país); Delineamento do estudo/instrumentos; Resultados e Conclusão (Quadro 1).
Quadro 1. Descrição dos estudos incluídos na revisão integrativa, segundo título, autor, periódico (ano), base de dados e tipo de pesquisa, Niterói, RJ, Brasil, 2017.

\begin{tabular}{|c|c|c|c|c|c|c|}
\hline $\begin{array}{l}N \\
0 \\
0\end{array}$ & Título & Autores & $\begin{array}{c}\text { Ano/País } \\
\text { Base de } \\
\text { dados / } \\
\text { Periódico / }\end{array}$ & $\begin{array}{c}\text { Delineame } \\
\text { nto } \\
\text { do estudo / } \\
\text { Instrument } \\
\text { os }\end{array}$ & Resultados & Conclusão \\
\hline 1 & $\begin{array}{c}\text { Percepção } \\
\text { de idosos } \\
\text { acerca das } \\
\text { atividades } \\
\text { desenvolvi } \\
\text { das no } \\
\text { hiperdial }\end{array}$ & $\begin{array}{c}\text { Reticena, } \\
\text { Kesley de } \\
\text { Oliveira; } \\
\text { Piolli, Kelly } \\
\text { Cristiane; } \\
\text { Carreira, } \\
\text { Lígia; } \\
\text { Marcon, } \\
\text { Sônia } \\
\text { Silva; } \\
\text { Sales, } \\
\text { Catarina } \\
\text { Aparecida. }\end{array}$ & $\begin{array}{c}\text { - } 2015 \\
\text { - Brasil } \\
\text { - LLLACS } \\
\text { - Revista } \\
\text { Mineira de } \\
\text { Enfermage } \\
\text { m (REME) }\end{array}$ & $\begin{array}{c}\text { Pesquisa } \\
\text { descritiva } \\
\text { exploratóri } \\
\text { a com } \\
\text { abordagem } \\
\text { qualitativa. } \\
\text { Pesquisa de } \\
\text { campo }\end{array}$ & \begin{tabular}{|l|}
-Atividades \\
educativas são \\
valorizadas; \\
-Associa-se o \\
acesso às \\
consultas e aos \\
medicamentos \\
às atividades \\
educativas; \\
-Revelaram-se \\
fragilidades no \\
desenvolviment \\
o das reuniôes \\
do HiperDia
\end{tabular} & $\begin{array}{l}\text { Os idosos que } \\
\text { participam das } \\
\text { atividades educativas } \\
\text { desenvolvidas no } \\
\text { HiperDia pela equipe } \\
\text { de estratégia saúde da } \\
\text { família percebem as } \\
\text { açóles educativas como } \\
\text { fonte de orientaçãoe } \\
\text { esclarecimento acerca } \\
\text { de sua saúde, } \\
\text { estimulando a } \\
\text { mudança de hábitos. E } \\
\text { associam essas } \\
\text { atividades educativas } \\
\text { ao acesso às consultas } \\
\text { e aos medicamentos, } \\
\text { sendo este o principal } \\
\text { evento motivador para }\end{array}$ \\
\hline & & & & & & $\begin{array}{l}\text { sua participação nas } \\
\text { mesmas. }\end{array}$ \\
\hline 2 & $\begin{array}{c}\text { Análise } \\
\text { biopolitica } \\
\text { do discurso } \\
\text { oficial } \\
\text { sobre } \\
\text { educaçãa } \\
\text { em saúde } \\
\text { para } \\
\text { pacientes } \\
\text { diabéticos } \\
\text { no Brasil|l| }\end{array}$ & $\begin{array}{c}\text { Melo, } \\
\text { Lucas } \\
\text { Pereira } \\
\text { de. }\end{array}$ & $\begin{array}{l}\text { - } 2013 \\
\text { - Brasil } \\
\text { - SClELLO } \\
\text { - Saúde e } \\
\text { Sociedade }\end{array}$ & $\begin{array}{c}\begin{array}{c}\text { Análise } \\
\text { documenta } \\
1\end{array} \\
\text { Qualitativa } \\
\begin{array}{c}\text { Revisão } \\
\text { bibliográfic } \\
\text { a }\end{array}\end{array}$ & 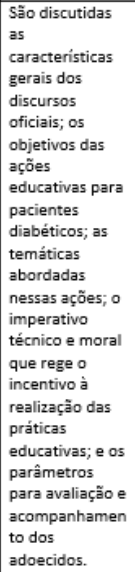 & $\begin{array}{l}\text { A análise do discurso } \\
\text { oficial possibilitou a } \\
\text { compreensão critica e a a } \\
\text { produção de } \\
\text { questionamento sobre } \\
\text { a complexa rede de } \\
\text { relaçôes dos objetos } \\
\text { postos em discurso nas } \\
\text { práticas educativas em } \\
\text { saúde para pacientes } \\
\text { diabéticos no Brasil. }\end{array}$ \\
\hline
\end{tabular}




\section{ARTIGO ORIGINAL}
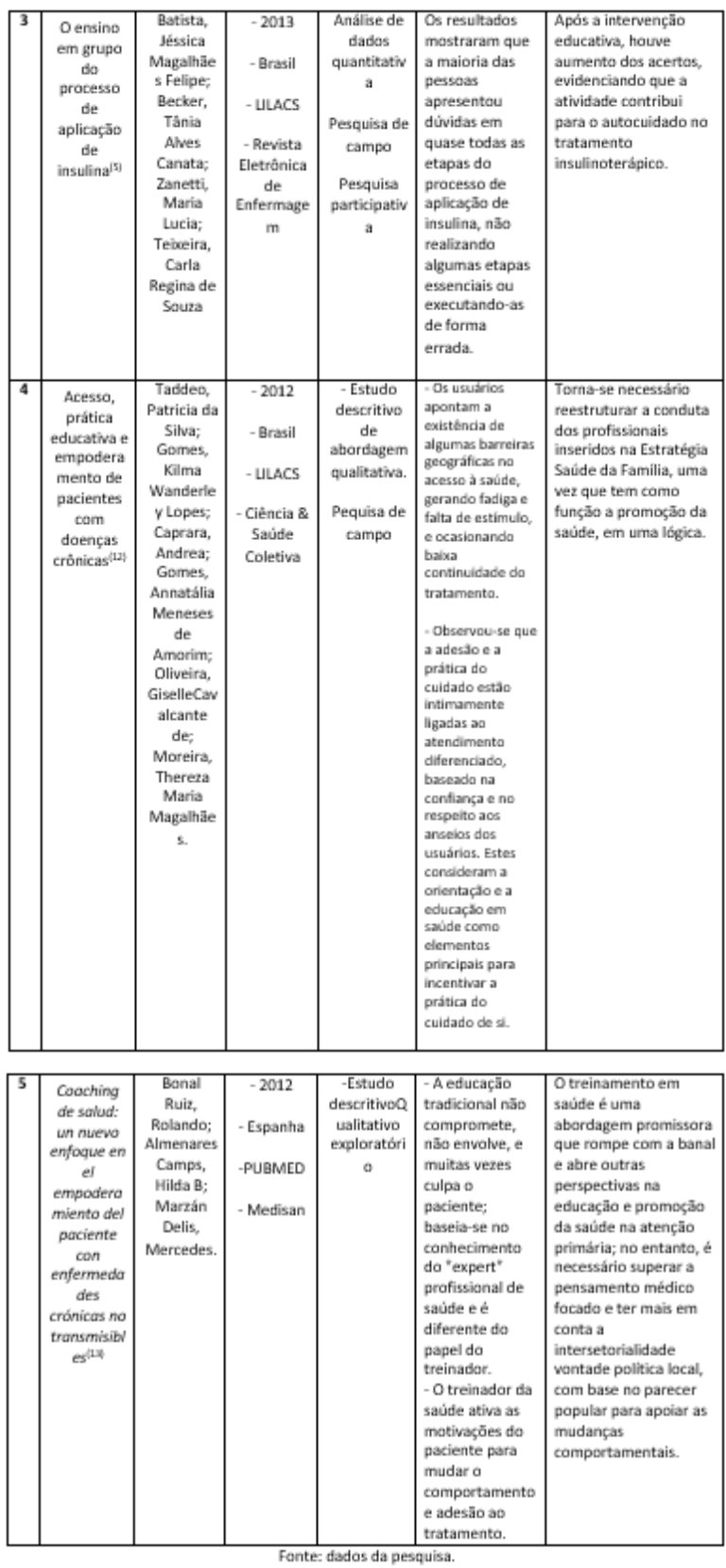

O resultado final da busca é composto por 5 (cinco) artigos, sendo 04 (80\%) na LILACS e 1 artigo (20\%) na PUBMED. Em maior número no idioma português, 04 artigos (80\%) e 01 artigo (20\%) em espanhol. Em relação ao ano de publicação, foram encontrados em ordem cronológica de publicação 02 artigos (40\%) em 2012, 02 artigos (40\%) em 2013, 01 artigo (20\%) em 2015, nos anos de 2014 e 2016 não foram encontrados artigos sobre a temática. Vale destacar que os 05 artigos pertencem a revistas distintas.

Quanto à natureza dos estudos, houve predominância de estudos com abordagem qualitativa, com 04 artigos (80\%), e 01 artigo (20\%) foi de abordagem quantitativa. Em relação aos tipos de estudos, 02 artigos (40\%) foram descritivos, 01 (20\%) descritivo exploratório, 01(20\%) de pesquisa participativa e $01(20 \%)$ não especificado. Identificou-se um maior número de pesquisas de campo em 04 artigos (80\%).

Ao avaliar a coleta de dados dos artigos pesquisados, podemos verificar que, dos artigos que fizeram pesquisa de campo, 03 (66,6\%) utilizaram como técnicas de produção de dados a entrevista aberta, a observação participante, roteiro e entrevista; 01 artigo $(33,33 \%)$ utilizou o instrumento checklist.

A partir da leitura dos artigos selecionados, procurando solucionar a questão de pesquisa, observamos que a valorização das atividades educativas apareceu nos 05 artigos (100\%), fragilidades no desenvolvimento dos encontros 40\% (02 artigos), educação verticalizada ainda presente em 02 artigos (40\%), acesso a medicamentos e consultas pelas atividades educativas 01 artigo (20\%).

A análise crítica e reflexiva da temática, metodologia, resultado e conclusão dos artigos selecionados permitiram a criação de 02 (duas) categorias: "A educação em saúde como forma de reorientação de hábitos de vida" e "Desafios na busca da superação do modelo biomédico".

\section{Discussão}

$\mathrm{Na}$ primeira metade do século passado, devido aos avanços da medicina e valorização da cultura curativa, a abordagem de educação em saúde não era vista como responsabilidade dos profissionais de saúde nem como forma de prevenção. Nessa época, pensava-se que esta prática educativa deveria pertencer aos educadores, pois esses eram considerados os únicos responsáveis pela reorientação de comportamentos individuais e coletivos ${ }^{(14-15)}$.

A partir da década de 1990, mudanças na estrutura do Sistema Único de Saúde no Brasil marcaram o 


\section{ARTIGO ORIGINAL}

início de um novo tempo para Saúde Pública. Com a criação da Estratégia de Saúde da Família, um novo paradigma surgiu, colocando o indivíduo e seu contexto social no centro das ações de saúde ${ }^{(16) \text {. }}$

Assim, os grupos de educação em saúde, visando melhoria na qualidade e aumento da expectativa de vida das pessoas que vivem com DCNT, passaram a ter maior destaque no modelo de atenção voltada para a família. Sendo assim, têm maior entendimento sobre o diálogo como estratégia de transformação da realidade, fornecendo instrumentos para conquista de autonomia e tomada de decisão sobre aspectos da vida ${ }^{(11-17-18)}$

Após leitura exaustiva dos artigos selecionados e análise crítica quanto a temática, metodologia e resultados dos estudos, foram construídas 02 (duas) categorias: A educação em saúde como forma de reorientação de hábitos de vida e Desafios para superação do modelo biomédico. Dos 05 (cinco) artigos, 03 (três) foram alocados na primeira categoria e 02 (dois) na segunda categoria.

A educação em saúde como forma de reorientação de hábitos de vida

É inegável a necessidade da utilização de grupos de educação em saúde como forma de reorientação das pessoas que vivem com DCNT. Os 05 artigos pesquisados abordaram as práticas de educação em saúde em grupo como forma de partilhar conhecimento e estimular o autocuidado.

$\mathrm{Em}$ pesquisa realizada em grupo educativo de pessoas que faziam uso de insulina, notou-se que após a realização dos grupos, os erros de armazenamento e administração diminuíram, contribuindo para a promoção da saúde e eficácia do tratamento. Tal atividade educativa era utilizada como forma de conhecer os problemas relacionados à auto-aplicação, e por meio da participação solucionar tais dificuldades, promovendo o autocuidado ${ }^{(11)}$.

A valorização do grupo se dá pelas orientações recebidas, pela ampliação do conhecimento e relevância do saber popular ${ }^{(11) .}$ Os grupos educativos são utilizados como espaço de discussão sobre a realidade de cada indivíduo e a valorização do conhecimento popular instrumentalizado pelo diálogo, fazendo com que o participante seja protagonista de seu processo de autocuidado(19).

Métodos participativos de educação em saúde são incentivados pelo Ministério da Saúde. Acredita-se que a valorização de saberes e práticas baseadas no diálogo fortalecem o vínculo do usuário com profissional, que deverá desempenhar o papel de dinamizador na construção de conhecimento a cerca das DCNTs $s^{(3-5-20-21)}$.

Os profissionais de saúde devem buscar novos caminhos para mudanças na realidade com os indivíduos, não há mais espaço para uma educação em saúde focada no discurso sobre a doença, devendo haver participação dos envolvidos na construção da prática educativa. Pode-se utilizar as ações educativas como forma de fomentar no indivíduo o real valor da saúde, instigando-os a serem co-responsáveis pelo processo saúdedoença(15-22).

Sobre as fragilidades no desenvolvimento das reuniões, podemos identificar que 02 artigos (40\%) abordam este tema. A dificuldade na locomoção e acesso às unidades de saúde restringem a possibilidade de adesão ao tratamento. Muitas vezes estes usuários chegam exaustos aos encontros e não ficam motivados em participar ${ }^{(12-}$ 23).

O provável insucesso de ações educativas surge das práticas discursivas utilizadas nos encontros, que muitas vezes inibem os participantes e não valorizam o conhecimento popular ${ }^{(6)}$.

Para que se obtenha êxito em grupos educativos, existem alguns princípios nos quais essas práticas devem estar pautadas: diálogo (ouvir o outro); tomar como ponto de partida do processo pedagógico 0 saber anterior das pessoas, acreditando que todos têm um conhecimento a partir de suas experiências e vivências; troca de experiências e construção de conhecimento entre o saber técnico e o saber popular, pressupondo que os diversos saberes são apenas diferentes, e não hierarquizados e que a experiência vale tanto quanto a teoria (24).

Dois dos artigos selecionados ressaltam que a 


\section{ARTIGO ORIGINAL}

prática educativa desenvolvida nos grupos de DCNT deve ser realizada de forma que o paciente participe dos encontros e haja uma reorientação do estilo de vida que ele possui. Como espaços de troca de saberes e escuta qualificada, o grupo educativo deve utilizar-se de métodos para minimizar anseios, sendo efetivo a fim de contribuir com o acesso e o tratamento ${ }^{(10,17)}$.

Três autores trazem um conceito sobre um método chamado "coaching da saúde", que consiste em por meio do treinamento de técnicas e abordagens sobre a doença, o usuário é "treinado" a praticar a autocuidado. A utilização desse método nos faz refletir sobre a real situação do usuário que será "treinado", contribuindo ou não para tomada de decisões em relação a sua saúde ${ }^{(13) .}$

Nota-se que somente 1 artigo (20\%) utilizou de maneira participativa o grupo como forma de construção de conhecimento. Há uma interação entre $o$ pesquisador e o grupo pesquisado, buscando-se uma horizontalidade nas ações e relações, almejando a transformação social vista como totalidade.

Desafios para superação do modelo biomédico Da totalidade dos artigos pesquisados, 2 artigos abordavam a discussão sobre o fato de que as pesquisas sobre DCNT têm se concentrado na investigação do indivíduo numa concepção biomédica do processo-saúde-doença.

Atualmente, as publicações têm se debruçado na fisiopatologia, terapêutica clínica e prevalência das DCNT, em detrimento das ações de promoção da saúde ${ }^{(25)}$.

A utilização de grupos educativos como forma de barganha de serviços oferecidos nas unidades de saúde não estimula mudanças e pouco repercutem na vida do usuário com vistas à promoção da saúde. $\mathrm{Na}$ medida em que as atividade de grupo são utilizadas para a comodidade do usuário, medidas assistencialistas, por meio de troca de receitas, rapidez de marcação de consultas e liberação de medicamentos, o objetivo do grupo educativo como modificador da realidade, é obsoleto e torna-se ineficaz ${ }^{(10)}$.

A priorização de assistência clínica ainda se faz presente nas unidades de saúde pública, ou seja, consultas e prescrições medicamentosas atuam como forma principal de intervenção $0^{(12) .}$

Ao estudar as práticas discursivas no processo de educação em saúde, as estratégias utilizadas são voltadas para promover a adesão do paciente, visando sua autonomia, porém, utilizando do poder de forma de convencimento ${ }^{(6)}$.

A realização de palestras como única forma de informação aos participantes do grupo é pouca motivadora. A utilização de metodologias não participativas não estimula um pensamento crítico, transformando os participantes do grupo em depósitos de informações ${ }^{(20) .}$

Os profissionais poucas vezes encontram outras maneiras de fazer educação em saúde sem ser impositiva e punitiva, devido ao fato de estarem arraigados no processo de trabalho hegemônico e assim, reproduz na prática o modelo prescritivo ${ }^{(6-26)}$ Há necessidade de um planejamento prévio para a realização de grupos educativos, os temas devem ser abordados de acordo com a necessidade e contexto das pessoas envolvidas ${ }^{(27) .} \mathrm{O}$ profissional enfermeiro tem participação fundamental no processo terapêutico. Ele é capaz de envolver o usuário com a utilização de técnicas, afim de obter tratamento adequado(28-29).Precisa conhecer as limitações e potencialidade de sua população assistida, deve procurar desenvolver ações que estimulem o autocuidado, "de forma a desenvolver essa ação, que não deve ser considerada somente como uma atividade a mais a ser realizada nos serviços de saúde, mas principalmente como prática que alicerça e reorienta toda a Atenção Primária à Saúde"(30).

Freire $^{(31),}$ em seu livro "Educação e Mudança", faz reflexões sobre o processo de educação e as mudanças sociais. $O$ saber deve ser construído entre o educador e o educando, de forma participativa e dialógica, e não com imposição de saberes ou práticas.

\section{Conclusão}

A partir desta pesquisa, podemos conhecer publicações on-line em formato de artigos científicos, no período de 2012 a 2016, sobre as atividades de grupos educativos em saúde com 


\section{ARTIGO ORIGINAL}

pessoas que vivem com DCNT e como os participantes enfrentam a doença. Foi percebida a necessidade de realização de atividades que fomentem a discussão sobre essa temática com grupos educativos.

Podemos citar como limitante na confecção deste estudo a escassez de referências sobre a repercussão desses grupos educativos acerca do autocuidado das pessoas que vivem com DCNT e a atuação do enfermeiro na realização desta estratégia de educação em saúde como forma de promoção da saúde.

As demais atribuições do dia-a-dia de trabalho dos profissionais de saúde acabam transformando os grupos em locais para depósito de informações. Há necessidade de realização dessas atividades, porém com planejamento adequado para utilização de novas estratégias. A repercussão dos grupos educativos deve ser mais discutida entre os profissionais enfermeiros e pessoas que vivem com DCNT.

A construção do conhecimento se dá de modo unilateral e autoritária, apesar dos artigos abordarem a importância da realização dos grupos educativos em saúde como forma de promoção da saúde. Atribui-se ainda ao grupo a realização de procedimentos técnicos, como aferição da pressão arterial, verificação da glicose capilar, e ainda revalidação de receitas médicas, distribuição de medicamentos e consulta médica ou de enfermagem. Isso faz com que os pacientes frequentem os grupos submetendo-se à posturas impositivas e autoritárias de profissionais, repercutindo na saúde dos mesmos.

Os grupos de educação em saúde são fundamentais no processo de reorientação de hábitos de vida de pessoas que vivem com DCNT. Porém, torna-se necessária a realização de atividades de educação permanente entre os profissionais, para que novas metodologias e estratégias de ensino em saúde sejam utilizadas.

Esperamos que este estudo contribua para a reflexão dos profissionais sobre as práticas educativas prestadas às pessoas com DCNT, a fim de fornecer instrumentos para o aperfeiçoamento das estratégias de educação em saúde, com objetivo de promover saúde e fortalecer a estrutura da atenção primária. A presente revisão integrativa forneceu um panorama geral das produções sobre o tema abordado, o qual trará para academia subsídios para construção de conhecimento os quais somados possam trazer avanços para as políticas de promoção da saúde.

\section{Referências}

1. Instituto Brasileiro de Geografia e Estatística (BR). Estudos e Pesquisas, Informação Demográfica e Socioeconômica número 24 . Projeção da População do Brasil por Sexo e Idade1980- 2050. [Internet]. Rio de Janeiro: IBGE; 2008. Disponível

em:http://www.ibge.gov.br/home/estatistica/popu lacao/projecao_da_populacao/2008/projecao.pdf\& gt.

2. Ministério da Saúde (BR). Plano de ações estratégicas para o enfrentamento das doenças crônicas nãotransmissíveis (DCNT) no Brasil 20112022. Brasília: Ministério da Saúde, 2011.

3. Ministério da Saúde (BR). Secretaria de Atenção à Saúde. Departamento de Atenção Básica. Estratégias para o cuidado da pessoa com doença crônica. Caderno 35. Departamento de Atenção Básica [Internet]. 2014 [acesso 10 set 2017]. Disponível em: http://dab.saude.gov.br/portaldab/biblioteca.php? conteudo=publicacoes/cab35.

4. Malta DC, Cezário AC, Moura L, Morais NOL, Silva JJB. A construção da vigilância e prevenção das doenças crônicas não transmissíveis no contexto do Sistema Único de Saúde. Epidemiol. Serv. Saúde [Internet]. 2006 Set [acesso 2018 Mar 13];15(3):47-65. Disponível em: http://scielo.iec.gov.br/scielo.php?script=sci_arttex t\&pid=S1679-49742006000300006.

5. Batista JMF, Becker TAC, Zanetti ML, Teixeira, CRS. O ensino em grupo do processo de aplicação de insulina. Revista Eletrônica de Enfermagem [Internet]. 2013 [acesso em 09 set 2017]; 15(1): 71-79. Disponível em: 


\section{ARTIGO ORIGINAL}

http://dxdoi.org/10.5216/rel.v15i1.16179.

6. Melo LP. Análise biopolítica do discurso oficial sobre educação em saúde para pacientes diabéticos no Brasil. Saúde Sociedade [Internet]. 2013 [acesso em 09 set 2017]; 22 (4): 1216-1225. Disponível em: http://dx.doi.org/10.1590/S010412902013000400022.

7. Malta DC, Merhy EE. O percurso da linha do cuidado sob a perspectiva das doenças crônicas não transmissíveis. Interface - Comunicação, Saúde e Educação [Internet]. 2010 [acesso em 13 março2018]; 14:593-605. Disponível em: http://dx.doi.org/10.1590/S1414-

32832010005000010 .

8. Santos ZMA, Lima HP. Tecnologia educativa em saúde na prevenção da hipertensão arterial em trabalhadores: análise das mudanças no estilo de vida. Texto \& Contexto - Enfermagem [Internet]. 2008 [acesso em 13 março 2018]; 17 (1): 90-97. Disponível em: http://dx.doi.org/10.1590/S010407072008000100010.

9. Mendes KDS, Silveira RCCP, Galvão CM. Revisão integrativa: método de pesquisa para a incorporação de evidências na saúde e na enfermagem. Texto \& Contexto - Enfermagem [Internet]. 2008 [acesso em 15 set 2017]; 17 (4): 758-764. Disponível em: http://dx.doi.org/10.1590/\$0104-

07072008000400018.

10. Souza MT, Silva MD, Carvalho R. Revisão Integrativa: o que é e como fazer. Einsten [Internet]. 2010 [acesso em 03 out 2017]; 8 (1): 102-106. Disponível em: www.scielo.br/pdf/eins/v8n1/pt_1679-4508-eins8-1-0102.pdf.

11. Reticena KO, Piolli KC, Carreira L, Maion SS, Sales CA. Percepção de idosos acerca das atividades desenvolvidas no hiperdia. Revista Mineira de Enfermagem [Internet]. 2015 [acesso em 09 set 2017]; 19 (2): 107-113. Disponível em: www.reme.org.br/exportar- pdf/1009/v19n2a09.pdf.

12. Taddeo PS, Gomes KWL, Caprara A, Gomes AMA, Oliveira GC, Moreira TMM. Acesso, prática e empoderamento de pacientes com doenças crônicas. Ciência \& Saúde Coletiva [Internet]. 2012 [acesso em 09 set 2017]; 17 (11):, 2923-2930. Disponível em: http://dx.doi.org/10.1590/S141381232012001100009 .

13. Ruiz RB, Campos HBA, Delis MM. Coaching de salud: um nuevo enfoque em elempodiramento Del paciente com enfermidades crônicas no transmisible. Medisan [Internet]. 2012 [acesso em 09 set 2017]; 16 (5): 773-785. Disponível em: http://scielo.sld.cu/scielo.php?script=sci_arttext\&p id=S1029-30192012000500014.

14. Acioli, S. A prática educativa como expressão do cuidado em Saúde Pública. Revista Brasileira de Enfermagem [Internet]. 2008 [acesso em 13 março 2018]; 61(1). 117-121. Disponível em: http://dx.doi.org/10.1590/S0034-

71672008000100019.

15. Reis TC, Figueiredo MFS, Souza e Souza LP, Silva JR, Amaral AKM, Messias RB, Leite MTS, Neto JFR. Educação em saúde: aspectos históricos no Brasil. J Health Scilnst [Internet]. 2013 [acesso em 09 set 2017]; 31 (2): 219-223. Disponível em: https://www.unip.br/presencial/comunicacao/.../ic s/.../V31_n2_2013_p219a223.pdf

16. Alves GG, Aerts D. As práticas educativas em saúde e a Estratégia de Saúde da Família. Ciência \& Saúde Coletiva [Internet]. 2011 [acesso em 09 set 2017]; 16 (1): 319-325. Disponível em: http://dx.doi.org/10.1590/S1413-

81232011000100034

17. Maia CCA, Rodrigues FG, Maia LA. Pelos Caminhos do SUS: Avanços e Perspectivas de uma Política. In: Souza MCMR, Horta NC Enfermagem em Saúde Coletiva. Teoria e Prática. Rio de Janeiro: Guanabara Koogan; 2012. p. 4-14.

18. Almeida, FA, Souza MCMR. Educação em 


\section{ARTIGO ORIGINAL}

saúde: Concepção e Prática no Cuidado de Enfermagem. In: Souza MCMR, Horta NC. Enfermagem em Saúde Coletiva. Teoria e Prática. Rio de Janeiro: Guanabara Koogan; 2012. p. 25-35

19. Medeiros MSN; Medeiros SM, costa RRO, Araujo MS, Medeiros KCMC. Estratégias de ensino e aprendizagem utilizadas no ensino de tópicos de atenção primária à saúde na graduação em enfermagem: uma revisão integrativa de literatura. Revista Enfermagem Atual [Internet]. 2017 [acesso em 26 março 2018]; 83; 78-85. Disponível em: http://revistaenfermagematual.com.br/21-11.php

20. Franco TAV, Silva JLL, Daher DV. Educação em saúde e pedagogia dialógica: uma reflexão sobre grupos educativos na atenção básica. Informe-se em Promoção da Saúde [Internet]. 2011 [acesso em 06 set 2017]; 7(2): 19-22. Disponível em: http://www.uff.br/promocaodasaude/grpos\%20ed .pdf

21. Mendonça FF, Nunes EFPA. Atividades participativas em grupos de educação em saúde para doentes crônicos. Cad. Saúde Coletiva [Internet]. 2014 [acesso em 09set 2017]; 22; 200204. Disponível em: http://10.1590/1414$462 \times 201400020014$

22. Azevedo PRA, Sousa MM, Sousa NF, Oliveira SHS. Ações de educação em saúde no contexto das doenças crôni-cas: revisão integrativa. Rev Fund Care Online [Internet] 2018. [acesso em 16 março 2018]; 10(1):260-267. Dispoínvel em: http://dx.doi.org/10.9789/2175-

5361.2018.v10i.260-267

23. Costa YF, Araújo OC, Almeida LBM. O papel educativo do enfermeiro na adesão ao tratamento da Hipertensão Arterial Sistêmica: revisão integrativa da literatura. O Mundo da Saúde [Internet]. 2014 [acesso em 09 set 2017]; 38(4): 473-481. Disponível em: http://10.15343/01047809.20143804473481
24. Sabóia VM, Valente GSC. A prática educativa em saúde nas consultas de enfermagem e vos encontros com grupos. Revista de Enfermagem Referência [Internet]. 2010 [acesso em 15 set 2017]; 2: 17-26. Diponível em: www.index-f.com/referencia/2010pdf/32-017.pdf

25. Silva LSS, Cotta RMM, Rosa COB. Estratégias de promoção da saúde e prevenção primária para enfrentamento das doenças crônicas: revisão sistemática. Revista Panamericana Salud Publica [Internet]. 2013 [acesso em 25 set 2017]; 34 (5): 343-350. Disponível em: https://www.scielosp.org/article/rpsp/2013.v34n5/ 343-350/

26. Lopes CR, Dalmolin IS, Durand MK, Rumor PCF, Heidemann ITSB, Koch C. Educação e cultura em saúde à luz de Paulo Freire. Revista de Enfermagem UFPE OnLine [Internet]. 2017 [acesso em 16 março 2018]; 11(12): 5122-8. Disponível em: https://doi.org/10.5205/1981-8963v11i12a25338p5122-5128-2017

27. Munari DB, Lucchese $R$, Medeiros $M$. Reflexões sobre o uso de atividades grupais na atenção a portadores de doenças crônicas. Ciência Cuidado Saúde [Internet]. 2009 [acesso em 09 set 2017]; 8: 148-154. Disponível em: http://doi:10.4025/ciencuidsaude.v8i0.9742

28. Berardinelli LMM, Guedes NAC, Clos C, Ramos P, Chaves ACS, Vieira, C. Produção do conhecimento em enfermagem acerca do empoderamento em situações crônicas de saúde. Revista enfermagem UERJ [Internet]. 2015 [acesso em 09 set 2017], 23 (3): 1414-1419. Disponível em: http://dx.doi.org/10.12957/reuerj.2015.16799

29. Silveira RES, Mendonça FTF, Santos AS, Filipe EMV. Estratégias de Educação em Saúde para idosos: experiência e desafios. Cultura de Los Cuidados [Internet]. 2015 [acesso em 02 out 2017]; 42: 154-163. Disponível em: http://dx.doi.org/10.14198/cuid.2015.42.140

30. Roecker S, Budó MLD, Marcon SS. Trabalho 


\section{ARTIGO ORIGINAL}

educativo do enfermeiro na Estratégia Saúde da Família: dificuldades e perspectivas de mudanças. RevEscEnferm USP [Internet] 2012 [acesso em 13 março 2018]; 46(3):641-9 Disponível em:http://dx.doi.org/10.1590/S0080-

62342012000300016

31. Freire P. Educação e mudança. Ed. Revisada e Atual. São Paulo: Paz e Terra. 2016. 